\title{
Perfusion MR Imaging Using a 3D Pulsed Continuous Arterial Spin-Labeling Method for Acute Cerebral Infarction Classified as Branch Atheromatous Disease Involving the Lenticulostriate Artery Territory
}

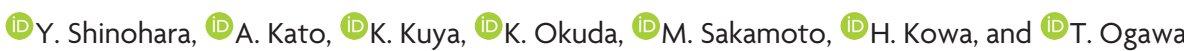

\begin{abstract}
BACKGROUND AND PURPOSE: Branch atheromatous disease is a stroke subtype considered a risk factor for early neurologic deterioration. Meanwhile, crossed cerebellar diaschisis is thought to be influenced by the degree and location of supratentorial perfusion abnormalities and is associated with the clinical outcome in the case of an ischemic stroke. In this article, our aim was to clarify the utility of using a whole-brain 3D pulsed continuous arterial spin-labeling method as an imaging biomarker for predicting neurologic severity in branch atheromatous disease.
\end{abstract}

MATERIALS AND METHODS: Twenty-three patients with branch atheromatous disease in the lenticulostriate artery territory were enrolled. All patients underwent MR imaging, including DWI, 3D-TOF-MRA, and 3D-arterial spin-labeling. We measured the asymmetry index of CBF in the affected area (branch atheromatous disease), the asymmetry index of the contralateral cerebellar hemisphere (crossed cerebellar diaschisis), and the DWI infarct volume in the lenticulostriate artery territory. We also compared each parameter with the initial NIHSS score with the Pearson correlation coefficient.

RESULTS: Among the 23 patients, we found no correlation between NIHSS score and the asymmetry index of CBF in the affected area (branch atheromatous disease) $(r=-0.027, P=.724)$, whereas the asymmetry index of the contralateral cerebellar hemisphere (crossed cerebellar diaschisis) and DWI infarct volumes were significantly correlated with NIHSS score $(r=0.515, P=.012 ; r=0.664, P=.001$, respectively).

CONCLUSIONS: In patients with branch atheromatous disease, 3D-arterial spin-labeling can detect crossed cerebellar diaschisis, which is correlated with the degree of neurologic severity.

ABBREVIATIONS: $\mathrm{Al}_{\mathrm{BAD}}=$ asymmetry index of the affected area (branch atheromatous disease); $\mathrm{Al}_{\mathrm{CCD}}=$ asymmetry index of the contralateral cerebellar hemisphere (crossed cerebellar diaschisis); $\mathrm{ASL}=$ arterial spin-labeling; $\mathrm{BAD}=$ branch atheromatous disease; $C C D=$ crossed cerebellar diaschisis; END = early neurologic deterioration; LSA = lenticulostriate artery; ref = reference

ntracranial branch atheromatous disease (BAD) is a stroke sub-
type that was first described in $1989 .{ }^{1}$ BAD was originally deter-
mined on the basis of pathologic findings of occlusion or stenosis
at the origin of a deep penetrating artery due to the presence of a
microatheroma or a junctional plaque. ${ }^{2,3}$ Representative vascular
territories of BAD include the lenticulostriate artery (LSA), the
pontine paramedian artery, and sometimes the anterior choroidal

Received November 19, 2016; accepted after revision March 27, 2017.

From the Division of Radiology (Y.S., A.K., K.K., T.O.), Department of Pathophysiological and Therapeutic Science, Faculty of Medicine; Division of Clinical Radiology (K.O.); Division of Neurosurgery (M.S.), Department of Brain and Neurosciences, Faculty of Medicine; and Division of Neurology (H.K.), Department of Brain and Neurosciences, Faculty of Medicine, Tottori University, Yonago, Japan.

Please address correspondence to Yuki Shinohara, MD, Division of Radiology, Department of Pathophysiological and Therapeutic Science, Faculty of Medicine, Tottori University, 36-1 Nishi-cho, Yonago 683-8504, Japan; e-mail:

shino-y@olive.plala.or.jp

http://dx.doi.org/10.3174/ajnr.A5247 artery. ${ }^{4,5}$ Previous studies have mentioned that BAD is strongly associated with early neurologic deterioration (END), and some patients with BAD may experience a worsening of neurologic deficits despite intensive medical treatment, resulting in a worse functional outcome. ${ }^{6-10}$ Although few pathologic studies for BAD have been reported, some articles have investigated its neuroradiologic characteristics, with MR imaging in particular. ${ }^{7-10}$

Arterial spin-labeling (ASL) is a noninvasive MR perfusion imaging technique that uses magnetically labeled blood as an intrinsic tracer. ${ }^{11-13}$ ASL permits a quantitative measurement of cerebral perfusion and repetitive acquisitions with independent labeling because it requires neither an injection of a contrast medium nor radiation exposure. ${ }^{11-13} 3 \mathrm{D}$-ASL is considered an advanced method for ASL and uses pulsed continuous arterial spinlabeling with a volumetric spiral fast spin-echo readout, which enables the acquisition of high signal-to-noise ratio and quantitative whole-brain perfusion images. ${ }^{13,14}$ 
Previous articles have suggested that 3D-ASL is useful for evaluating cerebral ischemia. ${ }^{12,15}$ To the best of our knowledge, no report has used ASL imaging findings to assess BAD, and a few articles have examined the neuroradiologic features of $\mathrm{BAD}$ that are related to END. ${ }^{6-8}$ In addition, some studies using ${ }^{15} \mathrm{O}$-positron-emission tomography and perfusion CT imaging have indicated that crossed cerebellar diaschisis (CCD) occurrence is influenced by the degree and location of the supratentorial perfusion abnormalities and is associated with the clinical outcome. ${ }^{16,17}$ Therefore, we hypothesized that perfusion abnormalities detected with 3D-ASL, including CCD, might be correlated with the degree of neurologic deterioration despite the small lesions found with BAD. The aim of our study is to clarify the utility of whole-brain 3D-ASL as an imaging biomarker for predicting the neurologic severity in BAD.

\section{MATERIALS AND METHODS \\ Patients}

This retrospective study analyzed data obtained from patients with acute cerebral infarctions who were admitted to our institution between October 2012 and November 2016. Patients were eligible if they met the following criteria: The NIHSS score on admission was obtained by stroke physicians; MR imaging, including 3D-ASL, DWI, and 3D-TOF-MRA, was performed; subtypes of intracranial BAD in the LSA region defined as a lesion of $\geq 15 \mathrm{~mm}$ in diameter and visible on $>3$ sections were observed on $\mathrm{DWI}^{9}$; there was no occlusion or severe stenosis ( $\left.\geq 50 \%\right)$ in the horizontal segment of the MCA on the MRA; and cardioembolism and large-artery atherosclerosis based on clinical features and other examinations, such as the pattern of symptom onset, resting electrocardiography, continuous electrocardiographic monitoring, transthoracic echocardiography, transesophageal echocardiography, and carotid ultrasonography, were excluded. ${ }^{18}$ Our institutional review board approved this study and waived the need for written informed consent because of its retrospective design.

\section{Imaging Examinations}

All MR imaging examinations were performed with a $3 \mathrm{~T} \mathrm{MR}$ imaging system (Discovery MR750w; GE Healthcare, Milwaukee, Wisconsin). For 3D-ASL, DWI, and MRA, the scanning parameters were the following: 3D-ASL: TR, $4554 \mathrm{~ms}$; TE, $10.7 \mathrm{~ms}$; FOV, $24 \mathrm{~cm}$; matrix, $512 \times 512$; section thickness, $4.0 \mathrm{~mm}$; postlabel delay, 1525 ms; acquisition time, 1 minute 31 seconds; DWI: TR, 7000 ms; TE, 10.7 ms; FOV, $21 \mathrm{~cm}$; matrix, $128 \times 128$; section thickness, $5.0 \mathrm{~mm}$; maximum $\mathrm{b}$ factor, $1000 \mathrm{~mm}^{2} / \mathrm{s}$; acquisition time, 56 seconds; MRA: TR, 20 ms; TE, $3.4 \mathrm{~ms}$; FOV, $20 \mathrm{~cm}$; matrix, $384 \times 224$; section thickness, $1.0 \mathrm{~mm}$; acquisition time, 4 minutes 36 seconds.

\section{Image Analysis}

3D-ASL maps were registered to DWIs by linear transformations (translation and rotation) by using SPM8 ((http://www.fil.ion. ucl.ac.uk/spm/software/spm8). BAD lesions in the section of the largest infarcted area of the LSA territory were outlined semiautomatically to measure the regional CBF (milliliter/100 $\mathrm{mL} / \mathrm{min}$ ) on 3D-ASL (ASL-BAD) according to the consensus of 2 neuroradiologists (Y.S. and A.K., with 13 and 5 years of experience in

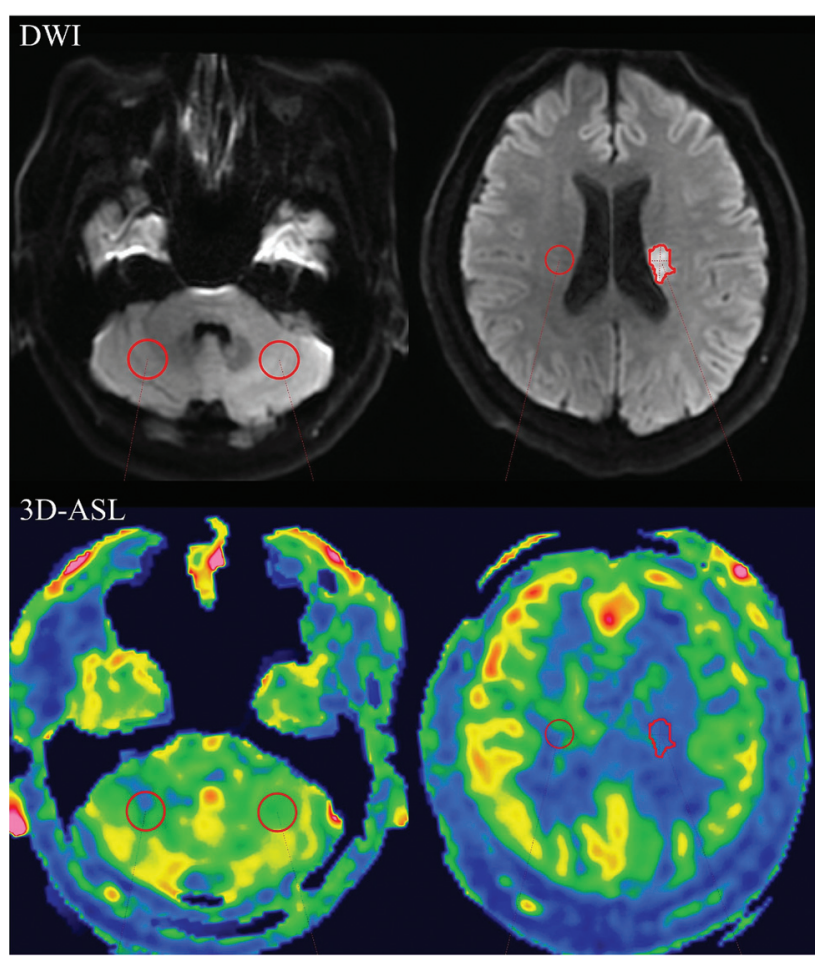

FIG 1. Representative sections of 3D-ASL images registered to the DWIs are shown with the locations of ROls outlined with red circles. On the section of the middle cerebellar peduncles and pons, elliptic ROls are marked on the bilateral cerebellum. On the section of the largest infarcted area, semiautomated ROI segmentation was performed in the affected area and an elliptic ROI was placed in the contralateral corona radiata.

diagnostic neuroradiology, respectively). ROIs with an elliptic shape were also marked on the contralateral corona radiata from the BAD lesions (ASL-BAD ${ }_{\text {reference }[\text { ref }]}$ ) and in the bilateral cerebellar hemispheres in the section of the middle cerebellar peduncles and pons (ASL-CCD and ASL-CCD ${ }_{\text {ref }}$ ) (Fig 1). The asymmetry index of the 3D-ASL based on the affected lesion was obtained as follows: asymmetry index of the affected area (branch atheromatous disease, $\mathrm{AI}_{\mathrm{BAD}}$ ) or asymmetry index of the contralateral cerebellar hemisphere (crossed cerebellar diaschisis, $\mathrm{AI}_{\mathrm{CCD}}$ ) = $\left(A S L-B_{\text {ref }}\right.$ or $-C_{\text {ref }}-\mathrm{ASL}-\mathrm{BAD}$ or $\left.-\mathrm{CCD}\right) /\left(\mathrm{ASL}-\mathrm{BAD}_{\text {ref }}\right.$ or $-\mathrm{CCD}_{\text {ref }}+\mathrm{ASL}-\mathrm{BAD}$ or $\left.-\mathrm{CCD}\right) \times 100(\%)$. DWI infarct volume measurements of $\mathrm{BAD}$ were performed with a semiautomated commercially available image-analysis program (AnalyzePro; AnalyzeDirect, Overland Park, Kansas).

\section{Statistical Analysis}

Linear regression analyses and Pearson correlation coefficients were used to compare the admission NIHSS score with each asymmetry index and DWI infarct volume. Each correlation was analyzed by using SPSS software (Version 23.0; IBM, Armonk, New York). $P<.05$ was statistically significant.

\section{RESULTS}

Twenty-three patients met our study criteria. Seventeen (73.9\%) patients were men, and 10 (43.5\%) lesions involved the left hemisphere. The mean patient age was $69.3 \pm 13.3$ years. The mean interval between symptom onset and MR imaging examination 

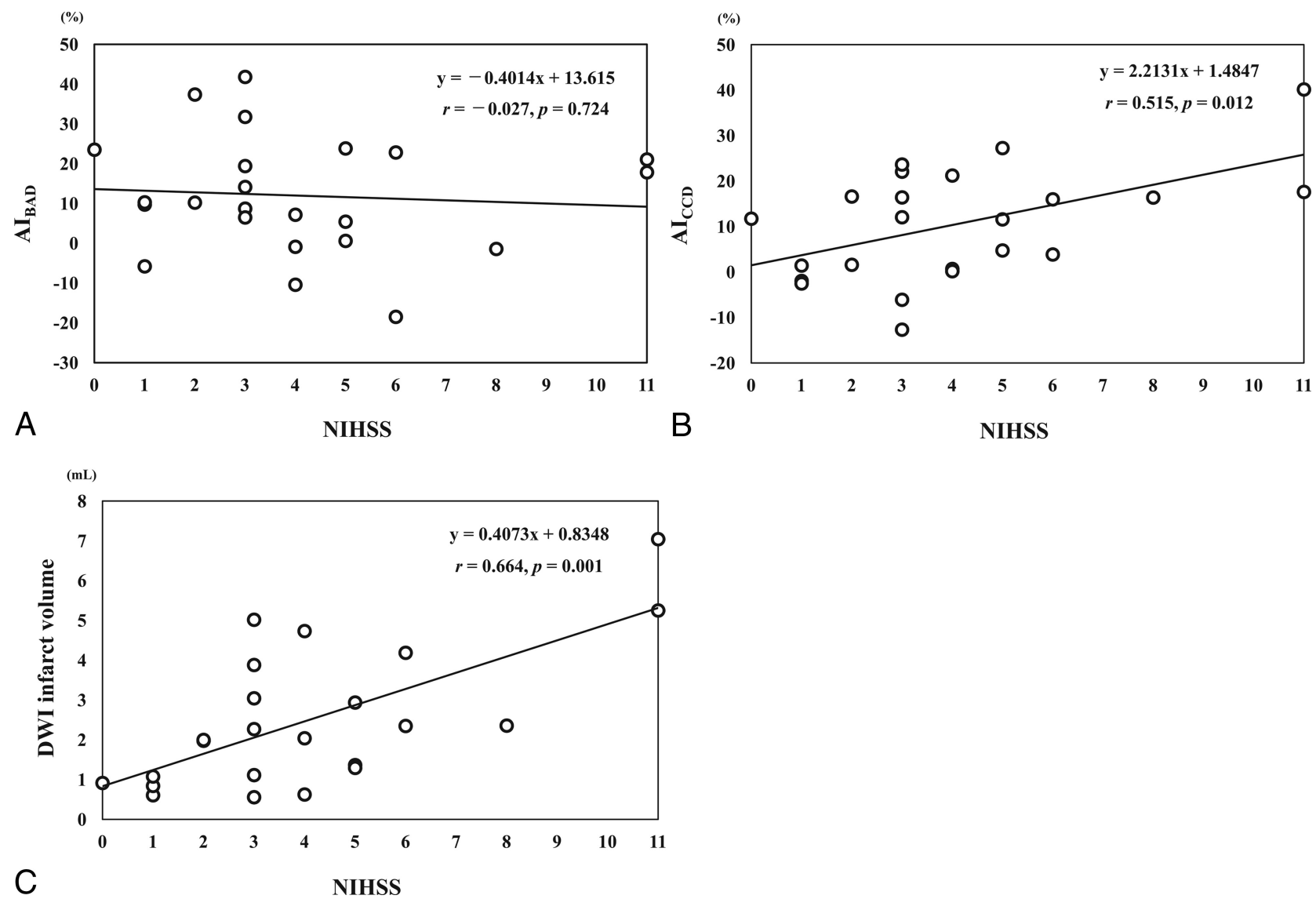

FIG 2. The relationships between admission NIHSS and the asymmetry index of the affected area (branch atheromatous disease, $\mathrm{Al}_{\mathrm{BAD}}$ ) and contralateral cerebellar hemisphere (crossed cerebellar diaschisis, $\mathrm{Al}_{\mathrm{CCD}}$ ) on 3D-ASL and DWI infarct volumes are shown. There was no significant correlation between admission NIHSS and $\mathrm{Al}_{\mathrm{BAD}}(A, r=-0.027, P=.724)$. Significant correlation was found between admission NIHSS and $\mathrm{Al}_{\mathrm{CCD}}(B, r=0.515 ; P=.012)$ and DWI volume $(C, r=0.664 ; P=.001)$.

was $40.6 \pm 37.6$ hours. The median admission NIHSS score was 3 (interquartile range, 2.5-5). The mean $\mathrm{AI}_{\mathrm{BAD}}$ and $\mathrm{AI}_{\mathrm{CCD}}$ were $12.0 \% \pm 14.5 \%$ and $10.5 \% \pm 12.1 \%$, respectively, and the mean DWI infarct volume was $2.50 \pm 1.72 \mathrm{~mL}$.

No significant correlation was found between NIHSS and $\mathrm{AI}_{\mathrm{BAD}}(r=-0.027, P=.724$, Fig $2 A)$. In contrast, we found a significant correlation between NIHSS and both $\mathrm{AI}_{\mathrm{CCD}}(r=$ $0.515, P=.012$, Fig $2 B)$ and DWI infarct volume $(r=0.664, P=$ .001 , Fig $2 C$ ). A demonstrable case is shown in Fig 3.

\section{DISCUSSION}

Our results showed that the CCD on 3D-ASL and DWI infarct volume are strongly correlated with the degree of neurologic severity in patients with BAD-related infarcts. END can often occur in patients with $\mathrm{BAD}$, and early management based on an accurate diagnosis is therefore needed in a clinical setting. These findings on 3D-ASL and DWI may represent important imaging biomarkers for predicting neurologic severity in the early stages of BAD.

Intracranial BAD was first described in 1989 as a new entity of atheromatous infarctions. ${ }^{1}$ The pathogenesis of BAD is considered an occlusion or stenosis at the origin of a deep penetrating artery due to the presence of a microatheroma or large parent artery plaque. ${ }^{2,10}$ Although the term BAD was originally presented on the basis of pathologic findings, few pathologic studies have been performed so far. ${ }^{3}$ In contrast, many neuroimaging studies of BAD have been performed, particularly with MR imaging. ${ }^{7-10}$ DWI shows comma-shaped restricted diffusion that extends to the basal surface of the parent artery in patients with $\mathrm{BAD}$, and noninvasive vessel imaging methods such as CTA or MRA usually reveal no or mild stenosis of the parent artery. ${ }^{19,20}$

Furthermore, BAD-related infarcts are considered predictive factors for END or progressive motor deficits. ${ }^{8}$ Yamamoto et $\mathrm{al}^{21}$ proposed that the severity of the neurologic status on admission (eg, initial NIHSS score) and preceding transient ischemic attacks could be predictors of END for LSA-type BAD. Meanwhile, a few reports have described the association between END and imaging findings of BAD. ${ }^{6-8}$ A previous study on BAD suggested that DWI findings associated with poor outcomes include a larger lesion size in a direction perpendicular to the axial section. ${ }^{22}$ This suggestion is compatible with our results in which the DWI infarct volume of BAD showed a favorable correlation with the initial NIHSS. Thus, an initial DWI may play an important role in estimating the neurologic severity of BAD-related infarcts.

3D-ASL is widely used as a noninvasive and repeatable wholebrain perfusion imaging method in clinical settings. ${ }^{11-15} 3 \mathrm{D}-\mathrm{ASL}$ enables acquisition of both the relative CBF near the affected lesions, including those associated with ischemia, tumors, or trauma, and the perfusion changes due to remote effects such as CCD. ${ }^{23-25} \mathrm{CCD}$ is a matched depression of blood flow and me- 

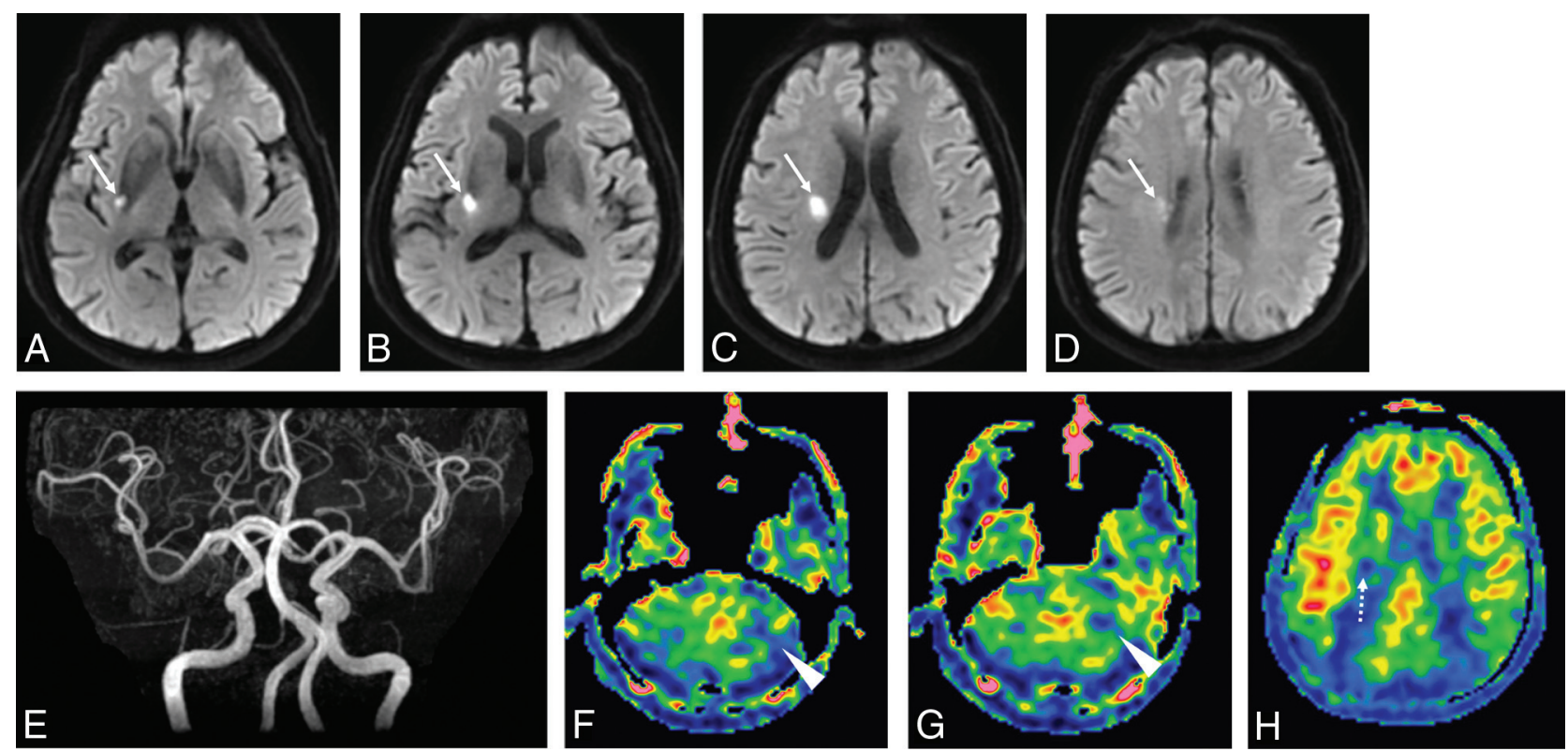

FIG 3. A 72 -year-old man with a right BAD-related infarction. The admission NIHSS score was 5 , and MR imaging was performed 24 hours after onset. $A-D$, DWls show high signal intensity from the posterior putamen to the corona radiata (white arrows). E, MRA reveals no stenosis or occlusion in the right MCA. $H$, The 3D-ASL shows hypoperfusion signal intensity in the right corona radiata, corresponding to the infarcted area (white dotted arrow). F and G, Hypoperfusion intensity in the left cerebellum (white arrowheads) can be observed, which indicates crossed cerebellar diaschisis due to the remote effect of the BAD-related infarction.

tabolism in the cerebellar hemisphere contralateral to a focal, supratentorial lesion and is a well-recognized phenomenon following cerebral infarctions. ${ }^{26}$ Previous articles have also indicated that asymmetry indices for CCD in 3D-ASL were well-correlated with those of SPECT. ${ }^{25}$ To the best of our knowledge, no reports have shown a relationship between 3D-ASL findings for BAD and neurologic status. In the present study, the CCD for BAD in 3DASL was much better correlated with the initial NIHSS score than the regional CBF of the 3D-ASL in the affected LSA region. Because the severity of neurologic status on admission is considered to be closely associated with the END in patients with $\mathrm{BAD},{ }^{21}$ the degree of initial CCD on 3D-ASL, as well as initial DWI infarct volume, may be predictors of END for LSA-type BAD.

CCDs may demonstrate good correlations with the initial NIHSS score due to the localization of LSA-type BAD, which usually affects the pyramidal tract of the corona radiata or the internal capsule so that secondary degenerative changes along the corticospinal tract may easily occur. According to a previous report with MR tractography, the degree of pyramidal tract involvement within the LSA territory infarction is strongly related to the severity of the stroke and functional recovery. ${ }^{27}$ Further studies with larger sample sizes are warranted to validate the relationship between CCD detected by 3D-ASL and the neurologic outcome, including follow-up by NIHSS.

Our study has several limitations. First, we examined the luminal patency with MRA and did not directly evaluate the parent artery wall. As previously mentioned, the pathogenesis of BAD is associated with proximal stenosis or occlusion of a penetrating artery due to a microatheroma or large parent artery plaque; and routine imaging techniques, such as MRA, are unable to visualize small vessel changes. However, recent studies showed that blackblood high-resolution MR imaging enables an evaluation of the intracranial artery vessel morphology, including the presence of plaques involving parent arteries. ${ }^{28}$ This capability could allow direct exploration of atheromatous small vessels and parent vessel lesions that cause BAD. Second, the interval from symptom onset to initial MR imaging examination varied widely among patients in this retrospective study. Finally, we only enrolled patients with LSA-type BAD. Patients with BAD involving other vascular territories, including the paramedian pontine artery, were not included because of the small sample size. A further prospective study that includes all types of BAD should be performed.

\section{CONCLUSIONS}

In patients with LSA-type BAD, the CCD detected by $3 \mathrm{D}$-ASL and DWI infarct volume was correlated with the severity of the neurologic status. Because the initial NIHSS score of BAD is considered a predictor of END and both 3D-ASL and DWI are noninvasive, repeatable, feasible sequences, they could be useful as radiologic imaging biomarkers for predicting the neurologic outcome in patients with BAD.

\section{REFERENCES}

1. Caplan LR. Intracranial branch atheromatous disease: a neglected, understudied, and underused concept. Neurology 1989;39:1246-50 CrossRef Medline

2. Fisher CM, Caplan LR. Basilar artery branch occlusion: a cause of pontine infarction. Neurology 1971;21:900-05 CrossRef Medline

3. Tatsumi S, Yamamoto T. An autopsied case of an apparent pontine branch atheromatous disease. Eur Neurol 2010;63:184-85 CrossRef Medline

4. Kwan MW, Mak W, Cheung RT, et al. Ischemic stroke related to intracranial branch atheromatous disease and comparison with large and small artery diseases. J Neurol Sci 2011;303:80-84 CrossRef Medline

5. Arboix A, Blanco-Rojas L, Martí-Vilalta JL. Advancements in understanding the mechanisms of symptomatic lacunar ischemic stroke:

AJNR Am J Neuroradiol 38:1550-54 Aug 2017 www.ajnr.org

1553 
translation of knowledge to prevention strategies. Expert Rev Neurother 2014;14:261-76 CrossRef Medline

6. Saia V, Pantoni L. Progressive stroke in pontine infarction. Acta Neurol Scand 2009;120:213-15 Medline

7. Nakase T, Yamamoto Y, Takagi M; Japan Branch Atheromatous Disease Registry Collaborators. The impact of diagnosing branch atheromatous disease for predicting prognosis. J Stroke Cerebrovasc Dis 2015;24:2423-28 CrossRef Medline

8. Yamamoto Y, Ohara T, Hamanaka M, et al. Characteristics of intracranial branch atheromatous disease and its association with progressive motor deficits. J Neurol Sci 2011;304:78-82 CrossRef Medline

9. Nakase T, Yoshioka S, Sasaki M, et al. Clinical evaluation of lacunar infarction and branch atheromatous disease. J Stroke Cerebrovasc Dis 2013;22:406-12 CrossRef Medline

10. Petrone L, Nannoni S, Del Bene A, et al. Branch atheromatous disease: a clinically meaningful, yet unproven concept. Cerebrovasc Dis 2016;41:87-95 CrossRef Medline

11. Deibler AR, Pollock JM, Kraft RA, et al. Arterial spin-labeling in routine clinical practice, part 1: technique and artifacts. AJNR Am J Neuroradiol 2008;29:1228-34 CrossRef Medline

12. Wolf RL, Detre JA. Clinical neuroimaging using arterial spin-labeled perfusion magnetic resonance imaging. Neurotherapeutics 2007;4:346-59 CrossRef Medline

13. Alsop DC, Detre JA. Multisection cerebral blood flow MR imaging with continuous arterial spin labeling. Radiology 1998;208:410-16 CrossRef Medline

14. Kimura H, Kabasawa H, Yonekura Y, et al. Cerebral perfusion measurements using continuous arterial spin labeling; accuracy and limits of a quantitative approach. International Congress Series 2004; 1265:238-47 CrossRef

15. Kimura H, Kado H, Koshimoto Y, et al. Multislice continuous arterial spin-labeled perfusion MRI in patients with chronic occlusive cerebrovascular disease: a correlative study with $\mathrm{CO}_{2}$ PET validation. J Magn Reson Imaging 2005;22:189-98 CrossRef Medline

16. Sobesky J, Thiel A, Ghaemi M, et al. Crossed cerebellar diaschisis in acute human stroke: a PET study of serial changes and response to supratentorial reperfusion. J Cereb Blood Flow Metab 2005;25: 1685-91 CrossRef Medline

17. Sommer WH, Bollwein C, Thierfelder KM, et al. Crossed cerebellar diaschisis in patients with acute middle cerebral artery infarction: occurrence and perfusion characteristics. J Cereb Blood Flow Metab 2016;36:743-54 CrossRef Medline

18. Adams HP Jr, Bendixen BH, Kappelle LJ, et al. Classification of subtype of acute ischemic stroke: definitions for use in a multicenter trial-TOAST. Trial of Org 10172 in Acute Stroke Treatment. Stroke 1993;24:35-41 CrossRef Medline

19. Bang OY. Intracranial atherosclerosis: current understanding and perspectives. J Stroke 2014;16:27-35 CrossRef Medline

20. Tamura A, Yamamoto Y, Nagakane Y, et al. The relationship between neurological worsening and lesion patterns in patients with acute middle cerebral artery stenosis. Cerebrovasc Dis 2013;35: 268-75 CrossRef Medline

21. Yamamoto Y, Ohara T, Hamanaka M, et al. Predictive factors for progressive motor deficits in penetrating artery infarctions in two different arterial territories. J Neurol Sci 2010;288:170-74 CrossRef Medline

22. Moriya S, Adachi T, Goto J, et al. Relationship between MRI findings and outcome in supratentorial branch atheromatous disease (BAD). Nosotchu 2006;28:504-09 CrossRef

23. Chen S, Guan M, Lian HJ, et al. Crossed cerebellar diaschisis detected by arterial spin-labeled perfusion magnetic resonance imaging in subacute ischemic stroke. J Stroke Cerebrovasc Dis 2014;23: 2378-83 CrossRef Medline

24. Strother MK, Buckingham C, Faraco CC, et al. Crossed cerebellar diaschisis after stroke identified noninvasively with cerebral blood flow-weighted arterial spin labeling MRI. Eur J Radiol 2016;85: 136-42 CrossRef Medline

25. Kang KM, Sohn CH, Kim BS, et al. Correlation of asymmetry indices measured by arterial spin-labeling MR imaging and SPECT in patients with crossed cerebellar diaschisis. AJNR Am J Neuroradiol 2015;36:1662-68 CrossRef Medline

26. Baron JC, Bousser MG, Comar D, et al. "Crossed cerebellar diaschisis" in human supratentorial brain infarction. Trans Am Neurol Assoc 1981;105:459-61 Medline

27. Konishi J, Yamada K, Kizu O, et al. MR tractography for the evaluation of functional recovery from lenticulostriate infarcts. Neurology 2005;64:108-13 CrossRef Medline

28. Chung JW, Kim BJ, Sohn CH, et al. Branch atheromatous plaque: a major cause of lacunar infarction (high-resolution MRI study). Cerebrovasc Dis Extra 2012;2:36-44 CrossRef Medline 